\title{
Transcatheter interventions for functional mitral regurgitation
}

\author{
William Z. Chancellor, Sarah A. Schubert, Gorav Ailawadi \\ Division of Thoracic and Cardiovascular Surgery, Department of Surgery, University of Virginia, Charlottesville, VA, USA \\ Correspondence to: Gorav Ailawadi, MD. Chief, Cardiothoracic Surgery, Department of Surgery, University of Virginia, PO Box 800679, \\ Charlottesville, VA 22903, USA. Email: Gorav@virginia.edu.
}

\begin{abstract}
Mitral regurgitation is considered the most prevalent valve disease in the United States and its incidence is increasing due to the aging population. Surgical mitral valve repair and replacement are effective at reducing regurgitation due to ischemia, but many patients with secondary mitral regurgitation do not undergo surgery because they are too high risk. Transcatheter devices that mimic surgical techniques are rapidly being developed to reduce the risks traditionally associated with surgical mitral valve repair and replacement. Despite the challenges posed by complex anatomy and multiple etiologies of mitral valve pathology, early results of transcatheter mitral repair and replacement indicate that they have the potential to increase the number of patients with secondary MR who are candidates for intervention.
\end{abstract}

Keywords: Mitral regurgitation; secondary mitral regurgitation; mitral valve repair; mitral valve replacement; transcatheter mitral valve intervention

Submitted Jul 20, 2018. Accepted for publication Aug 24, 2018.

doi: $10.21037 /$ acs.2018.09.01

View this article at: http://dx.doi.org/10.21037/acs.2018.09.01

\section{Introduction}

Mitral regurgitation (MR) is considered the most prevalent valve disease in the United States, affecting approximately $10 \%$ of the population older than 75 years old (1). While there are several etiologies of mitral valve disease, the most common is secondary, or functional MR (FMR), which is often caused by ischemic changes of the left ventricle (LV). Untreated MR is associated with life limiting symptoms of heart failure, increased hospitalizations and increased mortality (2-4). Therefore, current guidelines state that it is reasonable to consider surgery for severe FMR with symptoms or signs of $L V$ dysfunction (i.e., ejection fraction less than $60 \%$ or endsystolic diameter greater than $40 \mathrm{~mm}$ ) (5). However, there is evidence that many patients who meet these criteria are not offered surgery because they are not referred or are considered too high risk (6). A recent communitybased study from the Mayo Clinic in Rochester, $\mathrm{MN}$ reaffirms this lifelong undertreatment, which highlights the limitations of current options for the treatment of FMR (7). Given the increasing prevalence of secondary MR in the aging US population and a growing appreciation for its deleterious effects, the door is wide open for innovative therapies. Surgery will remain the gold standard for primary and secondary MR in appropriate patients for the foreseeable future, but device manufacturers are clamoring to complete feasibility studies for transcatheter devices and to begin enrolling randomized trials to treat patients who are not surgical candidates.

Catalyzed by the enormous success of transcatheter aortic valve replacement (TAVR), there are dozens of devices and interventions seeking approval for the treatment of MR. However, their path has not been straightforward because the etiology and presentation of MR is not as homogenous as aortic stenosis. From an anatomic stand-point, the mitral valve has a larger, less rigid annulus than the aortic valve, is more dynamic during the cardiac cycle and is more intimately associated with other structures, including the left circumflex coronary artery and left ventricular outflow tract (8). Fortunately, there are multiple treatment modalities being developed for transcatheter mitral valve repair (TMVr) and replacement (TMVR) with each of these challenges in mind. Given the variety of unique devices currently under development, a major issue going forward will be matching the best therapy, or combination of therapies, for each MR etiology, stage and for each patient's 

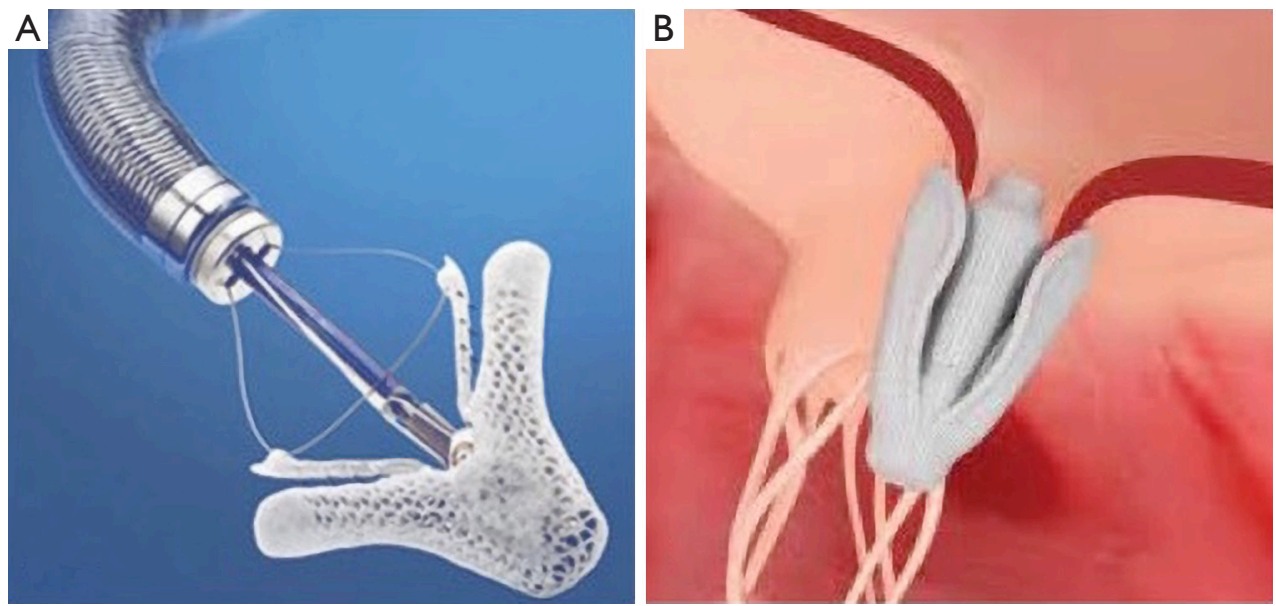

Figure 1 Percutaneous devices that achieve "edge-to-edge" mitral valve repair. (A) MitraClip (Abbott Vascular); (B) Pascal (Edwards Lifesciences).

unique anatomy.

\section{Repair}

At present, the only FDA-approved transcatheter device for the treatment of MR is MitraClip (Abbott Vascular). MitraClip is delivered transseptally and secures the anterior and posterior mitral leaflets together creating a doubleorifice valve with reduced surface area, thus reducing regurgitation. Its design is based on the surgical "edge-toedge repair", as described by Dr. Ottavio Alfieri, that is often used in conjunction with mitral annuloplasty to treat a variety of MR-causing lesions (9).

Based on the results of the EVEREST II trial, MitraClip was approved by the FDA in 2013 for use in patients with severe, symptomatic and degenerative MR thought to be at prohibitive risk for surgery (10). It had previously received CE mark approval in Europe in 2008, where it is also used for treatment of functional MR. Now, with more than 50,000 patients treated worldwide, MitraClip has cemented its place as a safe, reliable option that provides symptomatic improvement for patients who are not surgical candidates $(11,12)$. However, questions remain about its efficacy in patients with functional MR. Early, non-randomized studies that focused on high surgical risk patients demonstrated that MitraClip reduces regurgitation and improves New York Heart Association (NYHA) class in this patient population (13). A recent analysis of the STS/ACC TVT registry, designed to show the real-world, commercial results of MitraClip use, confirmed excellent procedural success rates and improved function, but demonstrated $49 \%$ cumulative incidence of death or repeat hospitalization for heart failure at 12 months in patients with FMR (14). At present, the structural heart community is eagerly awaiting results of the COAPT trial, which compares MitraClip to medical therapy alone for patients with clinically significant FMR (ClinicalTrials.gov. Unique identifier: NCT01626079). The results, which are expected late in 2018, will help determine if reducing the severity of FMR improves prognosis in patients already receiving optimal medical therapy (15).

MitraClip opened the door for the treatment of severe MR in high surgical risk patients, but elements of its design limit it to patients with a narrow range of anatomic lesions. These limitations were associated with lower technical success rates and increased reintervention in early studies (16,17). A recent redesign, MitraClip NT, allows for deeper insertion on valve leaflets, better grasping and easier retraction and regrasping. Taking a similar approach to mitral repair, Pascal (Edwards Life Sciences) is a transvenous, transseptal device that boasts a central spacer and wider, longer paddles that can be independently secured to the anterior and posterior valve leaflets (18) (Figure 1). Early experience with the device suggests that these features make it a better option for complex lesions, such as severe flail, pathology due to wide prolapse, or short posterior leaflets. Last year, Praz et al. reported outcomes of 23 compassionate use cases of severe MR $(>3+)$ in which technical procedural success was $96 \%$ and device success was $78 \%$ at 30 days (16). Their limited experience suggests 
A
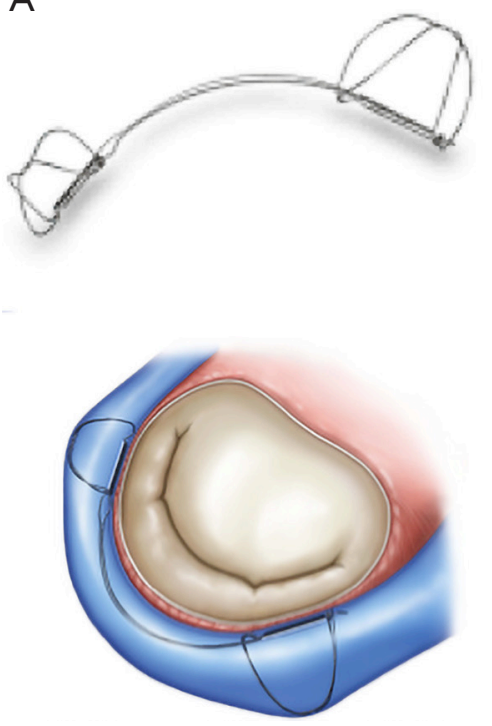

B
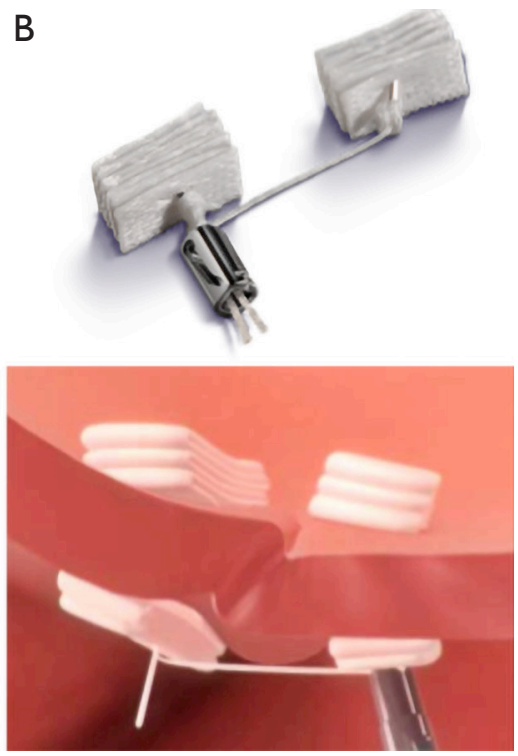

C
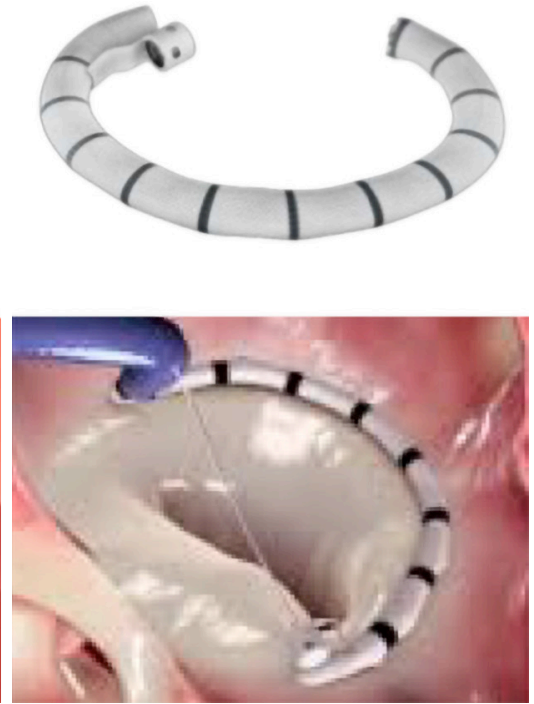

Figure 2 Transcatheter mitral annuloplasty devices. (A) Carillon (Cardiac Dimensions); (B) Mitralign (Mitralign Inc.); (C) Cardioband (Edwards Lifesciences).

we can expect Pascal will further expand the number of high-risk patients who are candidates for mitral valve repair to include those with complex leaflet pathology. Feasibility studies to determine the long-term safety and efficacy of Pascal are underway (ClinicalTrials.gov. Unique identifier: NCT03170349).

In terms of surgical repair, undersized annuloplasty is a mainstay for the treatment of FMR with a dilated mitral annulus. By decreasing the septal lateral length, annuloplasty increases coaptation of the mitral valve leaflets, thus reducing regurgitation (19). Currently, there are several devices under investigation that are seeking to replicate the technique of surgical annuloplasty through a percutaneous approach. Detailing the specifications and stage of development for every device is beyond the scope of this brief review. Instead, we hope to provide an overview of various techniques along with notable examples that are currently being studied (Figure 2).

Approaches to transcatheter mitral annuloplasty include direct methods that mirror surgical repair and novel, indirect methods. One particularly innovative technique is exemplified by the Carillon Mitral Contour System (Cardiac Dimensions), which consists of a fixed length, double anchor, nitinol device designed to be positioned within the coronary sinus (20). The benefit of utilizing the coronary sinus is that it is directly accessible from the femoral or jugular veins, which avoids a transseptal puncture. Results of the AMADEUS and TITAN trials demonstrated a significant reduction in functional MR grade and improved performance status in treated patients (21-23). As a result, Carillon is now CE mark approved and it is commercially available in some European countries. The randomized REDUCE FMR trial, which was designed to evaluate the effect of Carillon versus medical therapy alone in patients with suitable coronary sinus anatomy finished enrolling in January 2018 and results are expected later this year (ClinicalTrials.gov. Unique identifier: NCT02325830). The trial hopes to address lingering concerns regarding suitable anatomy for a coronary sinus-based device that include difficulty accessing the coronary sinus, atrial displacement of the device and potential compression of the left circumflex artery (24).

Direct annuloplasty techniques can be further divided based on their mechanism of annular reduction. For example, Mitralign (Mitralign Inc.) achieves annuloplasty through tissue plication using transannular pledgets at P1 and $\mathrm{P} 3$ that are cinched together and held in place with a stainless-steel locking device. An advantage of this technique is that it reduces annular dimension without eliminating the potential for future interventions (25). In an early feasibility study of 71 patients with FMR, the Mitralign device could not be deployed in 21 patients $(29.6 \%)$ due 

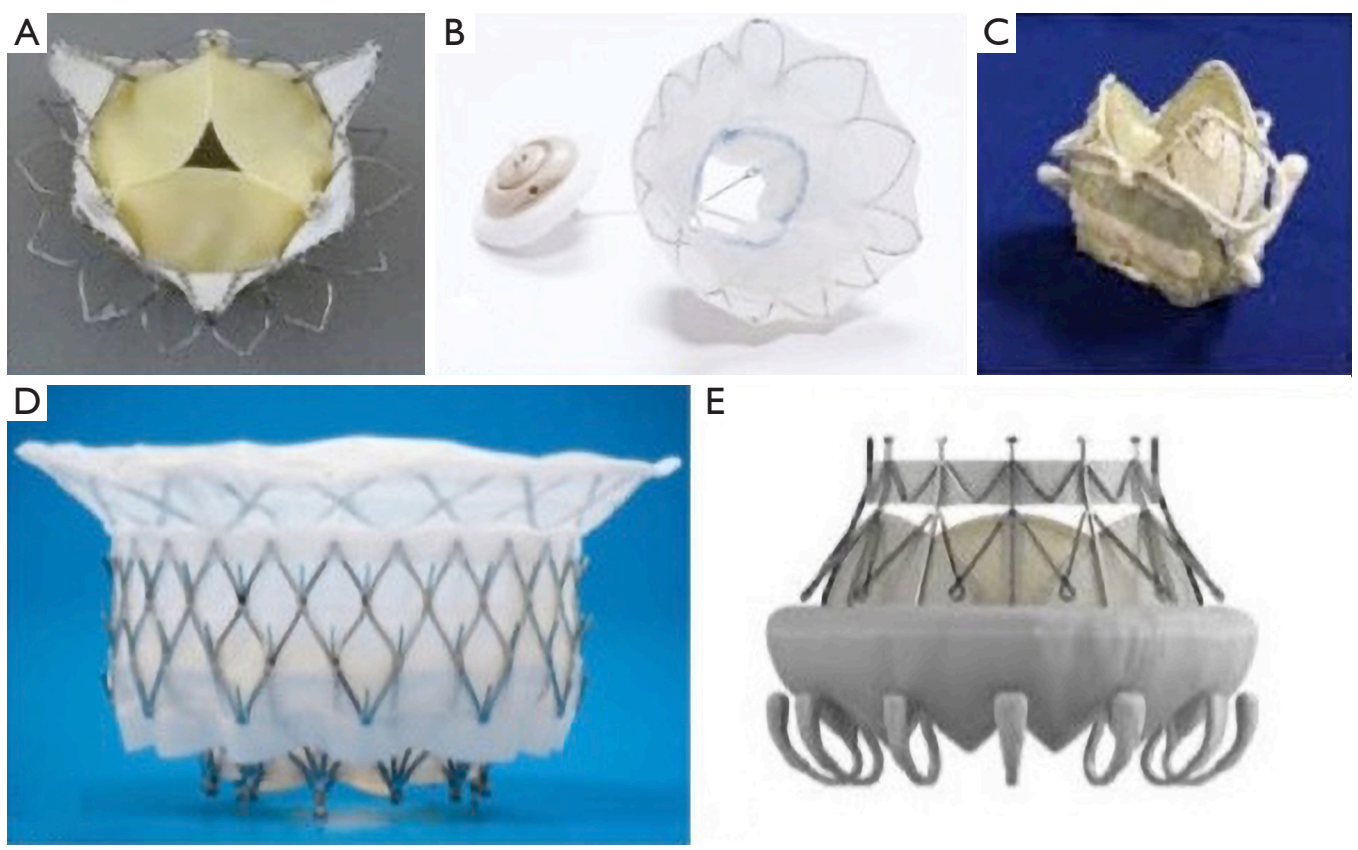

E

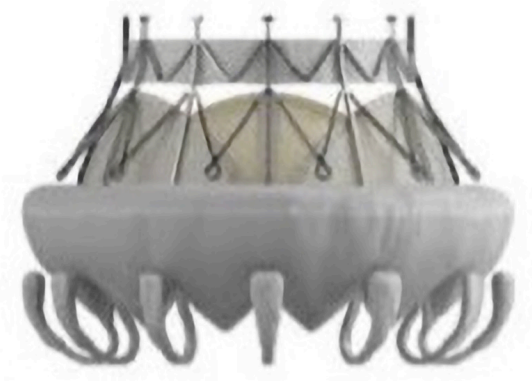

Figure 3 Transcatheter mitral valve replacement systems. (A) Tiara (Neovasc Inc.); (B) Tendyne (Abbott Vascular); (C) Caisson (LivaNova); (D) Intrepid (Medtronic); (E) CardiAQ (Edwards Lifesciences).

to anatomical or procedural challenges and was associated with MR reduction in just $50 \%$ of patients at 6 months. However, despite higher than expected persistent MR, patients exhibited beneficial left ventricular remodeling and symptom improvement (26).

Finally, the percutaneous devices that most closely resemble surgical repair techniques are direct annuloplasty devices like the Edwards Cardioband (Edwards Lifesciences). Cardioband is an incomplete, adjustable Dacron band delivered transseptally and implanted into the posterior annulus with stainless-steel anchors (27). It was approved for the treatment of FMR in Europe in 2015 and results of the European CE mark trial at 12 months were recently reported. The device was successfully inserted in 58 of 60 (97\%) high risk for surgery patients and MR was $<2+$ at 1 year in $95 \%$ of the patients who underwent follow-up transthoracic echocardiography. A randomized trial comparing Cardioband to guideline directed medical therapy is currently underway (ClinicalTrials.gov. Unique identifier: NCT03016975) (28,29).

While most transcatheter therapies for functional MR are seeking to replicate surgical techniques, there are devices at various stages of development that address the issue of ventricular remodeling instead of focusing on the mitral valve apparatus. Accucinch (Ancora Heart
Inc.), which is technically considered a direct annuloplasty device, was designed to correct and prevent left ventricular dilation in FMR. It is a retrograde system delivered via the femoral artery that is composed of 12-16 anchors, a cinch cable, spacers and a nitinol lock. The anchors attach to the ventricular free wall below the mitral annulus and leave the mitral apparatus untouched (30). Early data is limited, as safety and feasibility trials are ongoing (ClinicalTrials. gov. Unique identifier: NCT02153892), but the developer claims the device decreases MR and induces left ventricular remodeling (31).

\section{Replacement}

Mitral valve repair has long been the preferred surgical option for the treatment of regurgitation in appropriately selected patients. However, recent randomized evidence suggests mitral valve replacement is associated with decreased recurrence in the majority of patients with severe functional MR (32). These findings were published as the field of transcatheter mitral valve intervention began to gain momentum and they will likely be instrumental in its future. Similar to percutaneous repair, there are several transcatheter mitral valve replacement systems being developed simultaneously (Figure 3). Most are undergoing 
preclinical evaluation, but a few have moved into early feasibility studies. If these devices can mitigate the increased operative risk associated with surgical valve replacement while still offering freedom from MR recurrence, they may well become the preferred percutaneous option (33). However, the field is still in its infancy and there are many hurdles to overcome. Importantly, the mitral annulus is not nearly as rigid as a calcified aortic valve and is dynamic throughout the cardiac cycle, which makes anchoring and sealing a prosthesis difficult, especially since the valve will be exposed to $L V$ pressures.

The CardiAQ transcatheter mitral valve (Edwards Lifesciences) was the first transcatheter mitral valve implanted in a human (34). Like most of the current devices, it is composed of a self-expanding nitinol frame, but unlike most of the others it can be delivered transapically or transseptally. The CardiAQ valve is held in place by 12 atrial and 12 ventricular anchors and features a trileaflet design made of porcine pericardium (35). To date, implantation has reportedly been attempted for compassionate use in 13 patients with $92 \%$ technical success (36). Early feasibility trials are ongoing and a pilot study is planned but not currently enrolling (ClinicalTrials.gov. Unique identifiers: NCT02718001 and NCT2722551). Other devices that are enrolling early feasibility studies include the Caisson (LivaNova) and Tiara (Neovasc Inc.) mitral valve replacement systems (ClinicalTrials.gov. Unique identifiers: NCT02276547 and NCT02768402). Both valves are composed of trileaflet prostheses set in $\mathrm{D}$-shaped, self-expanding nitinol structures that are anchored above and below the mitral annulus (36,37). The D-shaped outer stent more closely approximates the native valve to allow improved anchoring and sealing. Like the CardiAQ valve, the Caisson valve has the advantage of being delivered transseptally.

Two TMVR systems, the Tendyne Mitral Valve System (Abbott Vascular) and the Intrepid TMVR system (Medtronic), have recently published promising early data. The Tendyne is a D-shaped, trileaflet prosthesis set in a self-expanding nitinol frame with a poly-terephthalate cuff for sealing above the annulus. Its most notable feature is an epicardial polyethylene tether designed to help it withstand ventricular pressures (38). Results of the Tendyne Global Feasibility Study were reported in 2017. Implantation was attempted in 30 patients with $93 \%$ procedural success. The patients were high risk for surgery with an average Society of Thoracic Surgeons Predicted Risk of Mortality (STS PROM) of 7.3 and nearly all (93\%) had 4+ MR at baseline.
Mortality at 1 year was $16 \%$ and 21 of 22 patients available for follow up reported no symptoms (39).

Results of the Intrepid TMVR system (Medtronic) Global Pilot Study results were also presented in late 2017. The study enrolled 50 patients and the procedural success rate was $96 \%$. Mortality at 30 days was $14 \%$, including 3 deaths attributable to apical access site bleeding and 1 death due to valve malposition. Median follow-up was 173 days and patients exhibited mild or no MR and significant improvements in symptoms and NYHA classification (40). While the functional results are certainly impressive, the Intrepid experience neatly sums up the current state of transcatheter mitral valve replacement: amazing potential to treat a difficult problem but with a few hurdles to overcome. Most notably, to improve its safety profile, TMVR will have to be done transseptally. The CardiAQ and Caisson devices have already demonstrated feasibility in this regard and others should follow suit. Besides the obvious operative risks of transapical deployment seen in the Intrepid trial, there is evidence that this approach may be detrimental for TAVR patients and it is likely more so in patients with existing $\mathrm{LV}$ dysfunction (41-43). Finally, strict anatomic criteria, such as having a sufficiently long left ventricular outflow tract to avoid high outflow gradients, may limit eligibility.

\section{Conclusions}

Overall, it is clear that the die has been cast and transcatheter mitral valve interventions will continue to improve and will increase the number of patients who are candidates for repair and replacement. However, the overwhelming number percutaneous options and limitations on anatomy means that it may take several years to enroll enough patients to draw meaningful conclusions from current and future studies. It also means that there is still plenty of room for all members of the structural heart team to embrace and become facile with these innovative therapies.

\section{Acknowledgements}

Funding: This work was supported by the National Heart, Lung, and Blood Institute (grant T32 HL007849).

\section{Footnote}

Conflicts of Interest: Dr. Ailawadi is a consultant for Abbott, Edwards, Medtronic, and Cephea. The other authors have 
no conflicts of interest to declare.

\section{References}

1. Nkomo VT, Gardin JM, Skelton TN, et al. Burden of valvular heart diseases: a population-based study. Lancet 2006;368:1005-11.

2. Grigioni F, Enriquez-Sarano M, Zehr KJ, et al. Ischemic mitral regurgitation: long-term outcome and prognostic implications with quantitative Doppler assessment. Circulation 2001;103:1759-64.

3. Enriquez-Sarano M, Avierinos JF, Messika-Zeitoun $\mathrm{D}$, et al. Quantitative determinants of the outcome of asymptomatic mitral regurgitation. $\mathrm{N}$ Engl J Med 2005;352:875-83.

4. Cioffi G, Tarantini L, De Feo S, et al. Functional mitral regurgitation predicts 1 -year mortality in elderly patients with systolic chronic heart failure. Eur J Heart Fail 2005;7:1112-7.

5. Nishimura RA, Otto CM, Bonow RO, et al. 2014 AHA/ ACC Guideline for the Management of Patients With Valvular Heart Disease: a report of the American College of Cardiology/American Heart Association Task Force on Practice Guidelines. Circulation 2014;129:e521-643.

6. Mirabel M, Iung B, Baron G, et al. What are the characteristics of patients with severe, symptomatic, mitral regurgitation who are denied surgery? Eur Heart J 2007;28:1358-65.

7. Dziadzko V, Clavel MA, Dziadzko M, et al. Outcome and undertreatment of mitral regurgitation: a community cohort study. Lancet 2018;391:960-9.

8. Sorajja P, Leon MB, Adams DH, et al. Transcatheter Therapy for Mitral Regurgitation Clinical Challenges and Potential Solutions. Circulation 2017;136:404-17.

9. De Bonis M, Lapenna E, Maisano F, et al. Long-term results ( $\leq 18$ years) of the edge-to-edge mitral valve repair without annuloplasty in degenerative mitral regurgitation: implications for the percutaneous approach. Circulation 2014;130:S19-24.

10. Feldman T, Foster E, Glower DD, et al. Percutaneous repair or surgery for mitral regurgitation. $\mathrm{N}$ Engl J Med 2011;364:1395-406.

11. Feldman T, Kar S, Elmariah S, et al. Randomized Comparison of Percutaneous Repair and Surgery for Mitral Regurgitation: 5-Year Results of EVEREST II. J Am Coll Cardiol 2015;66:2844-54.

12. Sorajja P, Mack M, Vemulapalli S, et al. Initial Experience with Commercial Transcatheter Mitral Valve Repair in the
United States. J Am Coll Cardiol 2016;67:1129-40.

13. Glower DD, Kar S, Trento A, et al. Percutaneous mitral valve repair for mitral regurgitation in high-risk patients: results of the EVEREST II study. J Am Coll Cardiol 2014;64:172-81.

14. Sorajja P, Vemulapalli S, Feldman T, et al. Outcomes with Transcatheter Mitral Valve Repair in the United States: An STS/ACC TVT Registry Report. J Am Coll Cardiol 2017;70:2315-27.

15. Asgar AW, Mack MJ, Stone GW. Secondary mitral regurgitation in heart failure: pathophysiology, prognosis, and therapeutic considerations. J Am Coll Cardiol 2015;65:1231-48.

16. Praz F, Spargias K, Chrissoheris M, et al. Compassionate use of the PASCAL transcatheter mitral valve repair system for patients with severe mitral regurgitation: a multicentre, prospective, observational, first-in-man study. Lancet 2017;390:773-80.

17. Orban M, Orban M, Lesevic H, et al. Predictors for longterm survival after transcatheter edge-to-edge mitral valve repair. J Interv Cardiol 2017;30:226-33.

18. Grasso C, Popolo Rubbio A. The PASCAL transcatheter mitral valve repair system for the treatment of mitral regurgitation: another piece to the puzzle of edge-to-edge technique. J Thorac Dis 2017;9:4856-9.

19. Bolling SF, Pagani FD, Deeb GM, et al. Intermediateterm outcome of mitral reconstruction in cardiomyopathy. $\mathrm{J}$ Thorac Cardiovasc Surg 1998;115:381-6; discussion 387-8.

20. Goldberg SL, Hammerstingl C. Coronary Sinus-Based Approach to Mitral Regurgitation. Interv Cardiol Clin 2016;5:93-100.

21. Schofer J, Siminiak T, Haude M, et al. Percutaneous mitral annuloplasty for functional mitral regurgitation: results of the CARILLON Mitral Annuloplasty Device European Union Study. Circulation 2009;120:326-33.

22. Siminiak T, Wu JC, Haude $M$, et al. Treatment of functional mitral regurgitation by percutaneous annuloplasty: results of the TITAN Trial. Eur J Heart Fail 2012;14:931-8.

23. Lipiecki J, Siminiak T, Sievert H, et al. Coronary sinusbased percutaneous annuloplasty as treatment for functional mitral regurgitation: the TITAN II trial. Open Heart 2016;3:e000411.

24. Feldman T, Cilingiroglu M. Percutaneous leaflet repair and annuloplasty for mitral regurgitation. J Am Coll Cardiol 2011;57:529-37.

25. Mandinov L, Asch FM, Grube E. Mitralign Percutaneous Annuloplasty System for the Treatment of Functional 
Mitral Regurgitation. European Cardiology Review 2011;6:67-70.

26. Nickenig G, Schueler R, Dager A, et al. Treatment of Chronic Functional Mitral Valve Regurgitation With a Percutaneous Annuloplasty System. J Am Coll Cardiol 2016;67:2927-36.

27. Maisano F, La Canna G, Latib A, et al. First-in-man transseptal implantation of a "surgical-like" mitral valve annuloplasty device for functional mitral regurgitation. JACC Cardiovasc Interv 2014;7:1326-8.

28. Nickenig G, Hammerstingl C, Schueler R, et al. Transcatheter Mitral Annuloplasty in Chronic Functional Mitral Regurgitation: 6-Month Results with the Cardioband Percutaneous Mitral Repair System. JACC Cardiovasc Interv 2016;9:2039-47.

29. Messika-Zeitoun D, Nickenig G, Latib A, et al. Transcatheter mitral valve repair for functional mitral regurgitation using the Cardioband system: 1 year outcomes. Eur Heart J 2018. [Epub ahead of print].

30. Taramasso M, Latib A. Percutaneous Mitral Annuloplasty. Interv Cardiol Clin 2016;5:101-7.

31. Promising findings to date. Ancora Heart. Accessed May 25, 2018. Available online: https://www.ancoraheart.com/ physicians/

32. Goldstein D, Moskowitz AJ, Gelijns AC, et al. Two-Year Outcomes of Surgical Treatment of Severe Ischemic Mitral Regurgitation. N Engl J Med 2016;374:344-53.

33. Rao C, Murphy MO, Saso S, et al. Mitral valve repair or replacement for ischaemic mitral regurgitation: a systematic review. Heart Lung Circ 2011;20:555-65.

34. Sondergaard L, De Backer O, Franzen OW, et al. Firstin-Human Case of Transfemoral CardiAQ Mitral Valve Implantation. Circ Cardiovasc Interv 2015;8:e002135.

35. Sondergaard L, Ussia GP, Dumonteil N, et al. The

Cite this article as: Chancellor WZ, Schubert SA, Ailawadi G. Transcatheter interventions for functional mitral regurgitation. Ann Cardiothorac Surg 2018;7(6):764-770. doi: 10.21037/ acs.2018.09.01
CardiAQ transcatheter mitral valve implantation system. EuroIntervention 2015;11 Suppl W:W76-7.

36. Regueiro A, Granada JF, Dagenais F, et al. Transcatheter Mitral Valve Replacement: Insights From Early Clinical Experience and Future Challenges. J Am Coll Cardiol 2017;69:2175-92.

37. Verheye S, Cheung A, Leon M, et al. The Tiara transcatheter mitral valve implantation system. EuroIntervention 2015;11 Suppl W:W71-2.

38. Perpetua EM, Reisman M. The Tendyne transcatheter mitral valve implantation system. EuroIntervention 2015;11 Suppl W:W78-9.

39. Muller DWM, Farivar RS, Jansz P, et al. Transcatheter Mitral Valve Replacement for Patients With Symptomatic Mitral Regurgitation: A Global Feasibility Trial. J Am Coll Cardiol 2017;69:381-91.

40. Bapat V, Rajagopal V, Meduri C, et al. Early Experience With New Transcatheter Mitral Valve Replacement. J Am Coll Cardiol 2018;71:12-21.

41. Ribeiro HB, Larose E, de la Paz Ricapito M, et al. Myocardial injury following transcatheter aortic valve implantation: insights from delayed-enhancement cardiovascular magnetic resonance. EuroIntervention 2015;11:205-13.

42. Ribeiro HB, Nombela-Franco L, Munoz-Garcia AJ, et al. Predictors and impact of myocardial injury after transcatheter aortic valve replacement: a multicenter registry. J Am Coll Cardiol 2015;66:2075-88.

43. Urena M, Webb JG, Eltchaninoff H, et al. Late cardiac death in patients undergoing transcatheter aortic valve replacement: incidence and predictors of advanced heart failure and sudden cardiac death. J Am Coll Cardiol 2015;65:437-48. 